\title{
Aetiological overlap between obsessive-compulsive and depressive symptoms: a longitudinal twin study in adolescents and adults
}

\author{
K. Bolhuis ${ }^{1}$ t, T. A. McAdams ${ }^{2}$ t, B. Monzani ${ }^{3}$, A. M. Gregory ${ }^{4}$, D. Mataix-Cols ${ }^{3}$, A. Stringaris ${ }^{1}$ \\ and T.C. Eley ${ }^{2 *}$ \\ ${ }^{1}$ Department of Child and Adolescent Psychiatry, King's College London, Institute of Psychiatry, London, UK \\ ${ }^{2}$ Social, Genetic and Developmental Psychiatry Centre, King's College London, Institute of Psychiatry, London, UK \\ ${ }^{3}$ Department of Psychosis Studies, Institute of Psychiatry, King's College London, Institute of Psychiatry, London, UK \\ ${ }^{4}$ Department of Psychology, Goldsmiths, University of London, London, UK
}

Background. Depression is commonly co-morbid with obsessive-compulsive disorder (OCD). However, it is unknown whether depression is a functional consequence of $\mathrm{OCD}$ or whether these disorders share a common genetic aetiology. This longitudinal twin study compared these two hypotheses.

Method. Data were drawn from a longitudinal sample of adolescent twins and siblings ( $n=2651$; Genesis 12-19 study) and from a cross-sectional sample of adult twins $(n=4920)$. The longitudinal phenotypic associations between OCD symptoms (OCS) and depressive symptoms were examined using a cross-lag model. Multivariate twin analyses were performed to explore the genetic and environmental contributions to the cross-sectional and longitudinal relationship between OCS and depressive symptoms.

Results. In the longitudinal phenotypic analyses, OCS at time 1 (wave 2 of the Genesis 12-19 study) predicted depressive symptoms at time 2 (wave 3 of the Genesis 12-19 study) to a similar extent to which depressive symptoms at time 1 predicted OCS at time 2. Cross-sectional twin analyses in both samples indicated that common genetic factors explained $52-65 \%$ of the phenotypic correlation between OCS and depressive symptoms. The proportion of the phenotypic correlation due to common non-shared environmental factors was considerably smaller (35\%). In the adolescent sample, the longitudinal association between OCS at time 1 and subsequent depressive symptoms was accounted for by the genetic association between OCS and depressive symptoms at time 1 . There was no significant environmental association between OCS and later depressive symptoms.

Conclusions. The present findings show that OCS and depressive symptoms co-occur primarily due to shared genetic factors and suggest that genetic, rather than environmental, effects account for the longitudinal relationship between OCS and depressive symptoms.

Received 4 January 2013; Revised 29 May 2013; Accepted 1 June 2013; First published online 7 August 2013

Key words: Depression, genetic correlation, heritability, obsessive-compulsive symptoms, pleiotropy.

\section{Introduction}

Obsessive-compulsive disorder (OCD) is characterized by distressing intrusive thoughts and time-consuming repetitive rituals. Although rare in very young children, OCD affects approximately $1-3 \%$ of the population (Flament et al. 1988; Heyman et al. 2001; Ruscio et al. 2010). OCD frequently co-occurs with anxiety, mood, impulse-control and substance use disorders (Heyman et al. 2006; Ruscio et al. 2010). Depressive

* Address for correspondence: T. C. Eley, Ph.D., The Social, Genetic and Developmental Psychiatry Centre, King's College London, Institute of Psychiatry, De Crespigny Park, London SE5 8AZ, UK.

(Email: thalia.eley@kcl.ac.uk)

+ These authors contributed as joint first authors. disorders are highly co-morbid in OCD (Heyman et al. 2006; Ruscio et al. 2010), with rates of depression ranging from $20 \%$ to $62 \%$ in paediatric samples with OCD (Douglass et al. 1995; Heyman et al. 2001; Ivarsson et al. 2008) and from $50 \%$ to $80 \%$ in adult samples with OCD (Rasmussen \& Eisen, 1992; Ruscio et al. 2010). This co-morbidity has been demonstrated in both epidemiological (Douglass et al. 1995; Fireman et al. 2001; Heyman et al. 2001; Ruscio et al. 2010) and clinical samples (Geller et al. 1996; Besiroglu et al. 2007; Ivarsson et al. 2008; Peris et al. 2010).

The reasons for the strong overlap between OCD and depression remain largely unknown. Two contrasting views regarding the relationship between OCD and depressive symptoms are apparent in the 
literature. One view is that depressive symptoms arise secondarily to the burden and impairment caused by obsessive-compulsive symptoms (OCS) (Storch et al. 2012). OCS lead to considerable functional impairment (Storch et al. 2010), familial turmoil (Peris et al. 2010) and peer problems (Storch et al. 2006) and, in turn, these factors are known to be associated with depression in their own right. This view is supported by data showing that depressive symptoms are associated with greater OCS severity and functional impairment (Peris et al. 2010; Storch et al. 2010; Krebs et al. 2013) and that depressive symptoms improve once OCD is successfully treated (Anholt et al. 2011). Furthermore, the prevalence of OCS during depressive episodes is $22-38 \%$ in patients with major depressive disorder (Gittleson, 1966; Kendell \& Discipio, 1970), a much lower prevalence compared with the high rate of depression in OCD.

The alternative view is that the relationship between OCS and depressive symptoms may be due to genetic overlap. OCD and depression co-aggregate in families (Nestadt et al. 2001; Carter et al. 2004; Hanna et al. 2011; Bienvenu et al. 2012; Goes et al. 2012) and it has been suggested that, similar to other anxiety disorders, genetic factors involved in the development of OCD may also be involved with the development of depressive disorders (Hettema, 2008). Indeed, the overlap between anxiety and depression is known to be due to shared genetic, rather than environmental, links, in both paediatric (Kendler et al. 2008; Zavos et al. 2010) and adult samples (Kendler \& Gardner, 2011). Given that OCD demonstrates a substantial degree of genetic overlap with other anxiety disorders (Tambs et al. 2009), one might expect that the overlap between OCS and depressive symptoms is also genetic in origin. Such an observation would be consistent with the 'genetic pleiotropy' hypothesis whereby generalist genes explain most of the overlap between various disorders while environmental effects explain most of the differences between disorders (Eley, 1997; Lahey et al. 2011). This genetic overlap between OCS and depressive symptoms could explain the overlap between their respective pathomechanisms, as both OCS and depressive symptoms are related to serotonergic brain disturbances and both respond to serotonergic medication (Insel, 1982; Zohar \& Insel, 1987).

This study explores the genetic and environmental influences on the overlap between OCS and depressive symptoms across the lifespan using a longitudinal twin study design. We predicted that this overlap would be primarily explained by genetic factors shared by the two phenotypes. If this were correct, we would expect strong genetic correlations between OCS and depressive symptoms in both cross-sectional and longitudinal analyses. The alternative hypothesis, that depressive symptoms arise as a result of the burden of OCS, could be described as an evocative gene-environment correlation, where the genetically influenced behaviour of the individual (i.e. OCS) evokes changes in the environment (e.g. conflict with parents) that in turn influence the likelihood of experiencing depressive symptoms. If this were the true process, we would expect high levels of overlap in the non-shared environmental influences on both OCS and later depressive symptoms (Jaffee \& Price, 2007; Kendler \& Baker, 2007).

\section{Method \\ Sample \\ Adolescent twin sample}

Data were drawn from the Genesis 12-19 (G1219) study, a longitudinal study of twins and siblings (McAdams et al. 2013). Initially 3640 adolescents participated. The present analyses focused on wave 2 and wave 3 (referred to hereafter as time 1 and 2, respectively), which on average took place 8 (range 0-24 months) and 33 months (range 24-60 months), respectively, after first contact. Data were available from 2651 individuals at time 1 (73\% of the original sample) and from 1597 individuals at time 2 (44\% of the original sample). The mean age of the sample was 15 years (range 12-21 years) at time 1 and 17 years (range 14-23 years) at time 2. More detailed information about the development and selection of the G1219 sample is available elsewhere (McAdams et al. 2013). Ethical approval for this study was provided by the Research Ethics Committees of the Institute of Psychiatry, King's College London and the South London and Maudsley NHS Trust.

\section{Adult twin sample}

Data were drawn from the TwinsUK adult twin registry (www.twinsuk.ac.uk), based at St Thomas' Hospital, London, which consists of a volunteer sample of approximately 10000 Caucasian adult twins, aged between 16 and 90 years (Moayyeri et al. 2013), ascertained from the general population. One time point was available in this sample. The present analyses focused on female twin pairs only [1319 monozygotic (MZ) pairs and 1141 dizygotic (DZ) pairs], as the relatively small number of male twin pairs $(n=318)$ and opposite-sex twin pairs $(n=66)$ did not allow investigation of sex differences. The mean age of the sample was 55 years (range 17-87 years). This study was approved by the St Thomas' Hospital Research Ethics Committee. 


\section{Measures}

\section{Adolescent sample}

In the adolescent sample, OCS were measured with the OCD subscale of the self-reported Spence Children's Anxiety Scale. This six-item scale was found to have a good internal consistency and adequate test-retest reliability (Spence, 1998). It has been shown to distinguish OCD cases from healthy controls and correlate well with a standard OCS measure (Whiteside et al. 2012).

Depressive symptoms were rated with the selfreported 13-item Short Mood and Feelings Questionnaire (SMFQ). At time 1, a four-point response format ('never' to 'always') was used to allow for discrimination at the lower end of the spectrum. The standard threepoint scale was used at time 2. The SMFQ has good internal consistency, test-retest stability and correlates well with other measures of depressed mood (Costello \& Angold, 1988; Costello et al. 1991; Angold et al. 1995).

\section{Adult sample}

In the adult sample, OCS were measured using the Obsessive-Compulsive Inventory-Revised (OCI-R), an 18-item five-point Likert scale. The OCI-R is a widely used measure for OCS and has been found to be a reliable, valid, clinically useful measure that correlates well with other OCS measures (Foa et al. 2002; Abramowitz \& Deacon, 2006).

Depressive symptoms were rated using a singleitem eight-point measure of depressed mood. The scale correlates strongly $(r>0.70)$ with the 21 -item Beck Depression Inventory and its empirically defined cut-offs have acceptable sensitivity and specificity (McKenzie \& Marks, 1999).

\section{Statistical analyses}

Phenotypic analyses

The cross-sectional associations between OCS and depressive symptoms were analysed using linear regression models corrected for sibling relatedness, performed in Stata version 10 for Windows (Stata Corp LP, USA). In the adolescent twin sample, the longitudinal relationship between OCS and depressive symptoms was studied using a cross-lagged model, corrected for sibling relatedness, using MPlus for Windows (Muthén \& Muthén, 1998).

\section{Genetic analyses}

Twin studies compare the phenotypic resemblance of MZ twins, who are $100 \%$ genetically identical, and DZ twins, who on average share $50 \%$ of their segregating genes. The effects of additive genetic (A), shared environmental ( $C$; environmental factors that make siblings alike) and non-shared environmental (E; environmental factors that make siblings different from one another, plus measurement error) factors on a trait can be estimated by comparing the within-pair correlations between $\mathrm{MZ}$ and DZ pairs. Genetic factors are assumed to play a role when $\mathrm{MZ}$ twin correlations are higher than DZ twin correlations. Shared environmental effects can be estimated by calculating the similarity between $\mathrm{MZ}$ pairs that is not due to genetic factors. Non-shared environmental effects can be calculated by subtracting the MZ correlations from 1 .

Twin analyses were performed using the program OpenMx (Boker et al. 2011). As is standard practice in quantitative genetic model fitting, residuals were taken prior to model fitting in order to account for the effects of age and sex on OCS and depressive symptoms. Variables in both samples were transformed using the Van der Waerden method to ensure normal distribution (Van der Waerden, 1933). Maximum-likelihood model-fitting analyses were undertaken to estimate the contribution of $\mathrm{A}, \mathrm{C}$ and $\mathrm{E}$ to the association between OCS and depressive symptoms (Neale \& Cardon, 1992). In the adolescent sample, the presence of quantitative sex differences for OCS and depressive symptoms was analysed. These analyses examine whether the extent to which genetic, shared environmental and non-shared environmental factors contribute to the total variance is different for males and females. We also tested for the presence of total variance differences between male and females (a scalar model). Submodels were assessed with $\chi^{2}$ difference tests and Akaike's information criterion (AIC) to test whether constraining the effects of $\mathrm{A}, \mathrm{C}$ or both to zero would result in a significant reduction in fit, with lower values indicating a better model fit (Neale \& Cardon, 1992).

Bivariate twin analyses were performed to explore the origins of the cross-sectional relationship between OCS and depressive symptoms in both samples. The proportion of the phenotypic overlap that can be explained through genetic and environmental factors was calculated. The sum of these proportions adds up to 1 .

The longitudinal relationship between OCS and depressive symptoms was explored in the adolescent sample. A Cholesky decomposition was chosen, in which covariation between variables in additive genetic (A), shared environmental (C) and non-shared environmental factors (E) was examined (Fig. 1). In this model, analyses were corrected for the crosssectional relationship between OCS and depressive symptoms at time 1 by ordering the variables in such a way that depressive symptoms at time 1 were placed before OCS scores at time 1 (Fig. 1). A Cholesky 


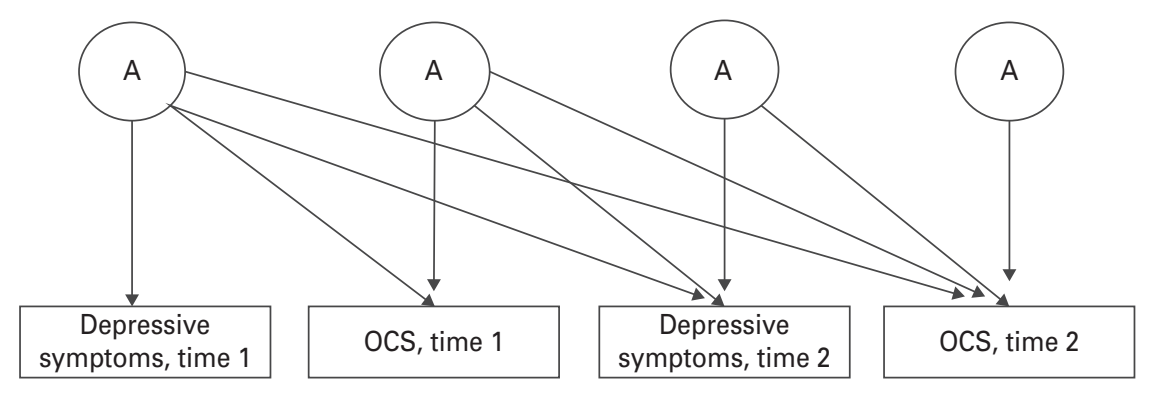

Fig. 1. Cholesky decomposition for obsessive-compulsive disorder symptoms (OCS) and depressive symptoms at time 1 and time 2 in the adolescent sample. The first factor for additive genetic effects (A) accounts for the genetic variance that is common to all four variables. The second factor accounts for the remaining three variables not accounted for by the first factor, and so on. The last factor accounts for the remaining variance in the final variable that is not shared with any other variable in this model.

decomposition was chosen over a single-factor or cross-lag approach in which, respectively, a single latent factor explains the covariance between variables and where the covariation between variables is decomposed in the contributive effects of A, C and E (Neale \& Cardon, 1992), as it allowed us to test the two opposing hypotheses in a single model. Models were fitted to generate genetic and environmental path estimates between the four variables (Fig. 1).

As is standard practice in quantitative genetic studies, standardized path estimates were presented in figures, whereas tables showed the standardized squared path estimate which are also referred to at times as variance components. The values of the latter add up to 1 .

\section{Results}

\section{Demographic characteristics}

Online Supplementary Table S1 presents the means, standard deviations and regression coefficients for the effects of sex and age on OCS and depressive symptoms in both samples. Online Supplementary Table S2 presents the within-trait twin correlations of both traits in the two samples. At both time points in the adolescent sample, depressive symptom scores were significantly higher in females than in males, and at time 1 , though not at time 2 , depressive symptom scores were associated with an older age. In the adolescent sample, OCS scores were unrelated to age or sex. In the adult sample, OCS and depressive symptoms were significantly associated with a younger age.

\section{Phenotypic findings}

Cross-sectional associations between OCS and depressive symptoms

In the adolescent sample, OCS were significantly associated with depressive symptoms at both time 1 $[\beta=0.51, \quad 95 \%$ confidence interval (CI) 0.46-0.55, $p<0.001]$ and time $2 \quad(\beta=0.46,95 \%$ CI $0.40-0.52$, $p<0.001)$. This was also true for the adult sample $(\beta=0.41,95 \%$ CI $0.34-0.48, p<0.001)$.

Longitudinal associations between OCS and depressive symptoms

The cross-lag model of the longitudinal associations between OCS and depressive symptoms (Fig. 2) shows that the path coefficient from OCS at time 1 to depressive symptoms at time $2 \quad \beta=0.08,95 \%$ CI 0.02-0.16) is similar to the pathway from depressive symptoms at time 1 to OCS at time $2(\beta=0.07,95 \%$ CI 0.01-0.15). Within-trait longitudinal, and cross-trait within-time pathways were all substantial (range $\beta=0.39-0.51$ ).

\section{Genetic findings}

Univariate analyses of OCS and depressive symptoms

In the adolescent twin sample, scalar sex-limitation models provided the best fit to the data for OCS at time 2 and depressive symptoms at both time points. In these models estimates of genetic and environmental effects were constrained to be the same for males and females. A scalar was included to account for variance differences between the sexes. That is, whilst there was greater variance in female scores, genetic and environmental factors contributed to the variance in OCS and depressive symptoms in the same manner in males and females. A no sex-differences model without a scalar provided the best fit for OCS at time 2. Shared environment effects could be dropped from each of the four models fitted to the adolescent data. In the adult sample, the best fit for OCS was the ACE model, whereas for depressive symptoms this was the AE model. Univariate model-fitting results 
Table 1. Results from the cross-sectional bivariate correlated-factor models for the cross-sectional association between OCS and depressive symptoms, for all three age groups

\begin{tabular}{|c|c|c|c|}
\hline & $\begin{array}{l}\text { Adolescent sample, } \\
\text { time } 1(95 \% \mathrm{CI})\end{array}$ & $\begin{array}{l}\text { Adolescent sample, } \\
\text { time } 2(95 \% \mathrm{CI})\end{array}$ & $\begin{array}{l}\text { Adult sample } \\
(95 \% \mathrm{CI})\end{array}$ \\
\hline Phenotypic correlation & $0.53(0.48$ to 0.55$)$ & $0.47(0.43-0.51)$ & $0.44(0.42-0.47)$ \\
\hline $\begin{array}{l}\text { Proportion of the phenotypic correlation due to } \\
\text { genetic factors }(\mathrm{A})\end{array}$ & $0.52(0.26$ to 0.85$)$ & $0.65(0.50-0.78)$ & $0.65(0.57-0.73)$ \\
\hline $\begin{array}{l}\text { Proportion of the phenotypic correlation due to } \\
\text { shared environmental factors }(\mathrm{C})\end{array}$ & $0.13(-0.10$ to 0.32$)$ & - & - \\
\hline $\begin{array}{l}\text { Proportion of the phenotypic correlation due to } \\
\text { non-shared environmental factors (E) }\end{array}$ & $0.35(0.21$ to 0.47$)$ & $0.35(0.22-0.50)$ & $0.35(0.27-0.43)$ \\
\hline
\end{tabular}

OCS, Obsessive-compulsive disorder symptoms; CI, confidence interval.

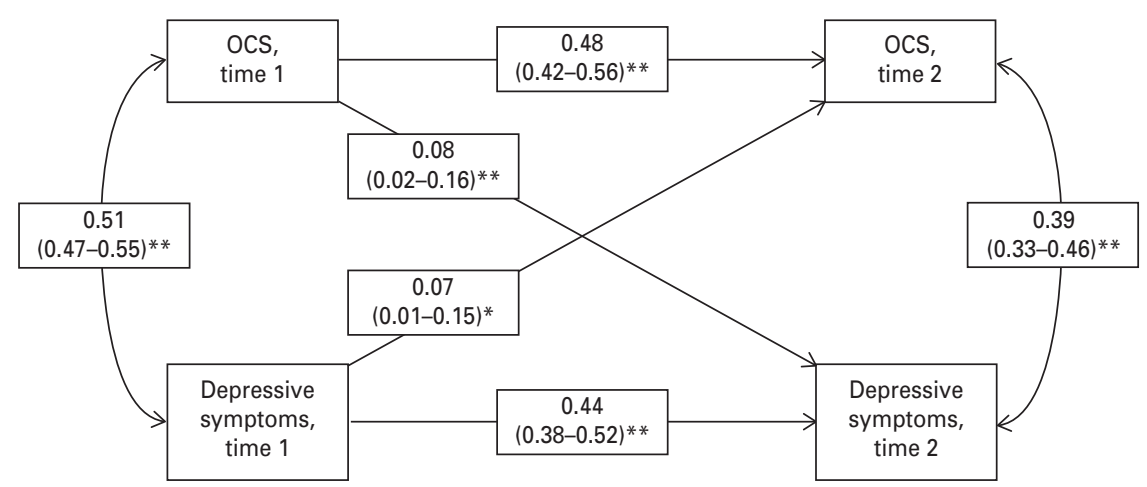

Fig. 2. Cross-lag model of the standardized cross-sectional and longitudinal phenotypic associations between obsessive-compulsive disorder symptoms (OCS) and depressive symptoms in the adolescent twin sample. The longitudinal path coefficients were controlled for the effects of depressive symptoms at time 1 and OCS at time 1, and for sibling relatedness. Values in parentheses are $95 \%$ confidence intervals. ${ }^{*} p<0.05,{ }^{* *} p<0.01$.

are reported in online Supplementary Table S3. Additive genetic effects on OCS and depressive symptoms were moderate in both samples (online Supplementary Tables S1 and S2).

\section{Cross-sectional genetic and environmental associations between OCS and depressive symptoms}

The genetic and environmental influences on the crosssectional relationship between OCS and depressive symptoms were analysed using a correlated-factors approach. Bivariate model-fitting analyses are reported in online Supplementary Table S4. An AE model was used for time 2 of the adolescent sample and for the adult sample. An ACE model was used for time 1 of the adolescent sample. In the adolescent sample at time 1, shared environmental factors did not play a significant role on the individual traits, but were significant in the association between traits. In the adult sample the opposite was the case: shared environmental factors had a significant effect on OCS but did not play a significant role in the association between OCS and depressive symptoms. However, overall, shared environmental factors were of marginal influence in both samples and had very limited effects compared with the genetic and non-shared environmental factors. Table 1 shows the cross-sectional phenotypic correlations between OCS and depressive symptoms in both samples. In addition, the proportion of each of these correlations due to genetic, shared environmental and non-shared environmental factors is given. Fig. 3 presents the unsquared standardized path estimates of genetic and environmental influences on the cross-sectional association between OCS and depressive symptoms for the different age groups. It also presents the genetic, shared environmental and non-shared environmental correlations between OCS and depressive symptoms in both samples.

Fig. 3 demonstrates that for all three age groups (range 12-87 years), OCS and depressive symptoms are highly genetically correlated (range 0.71-0.86). 

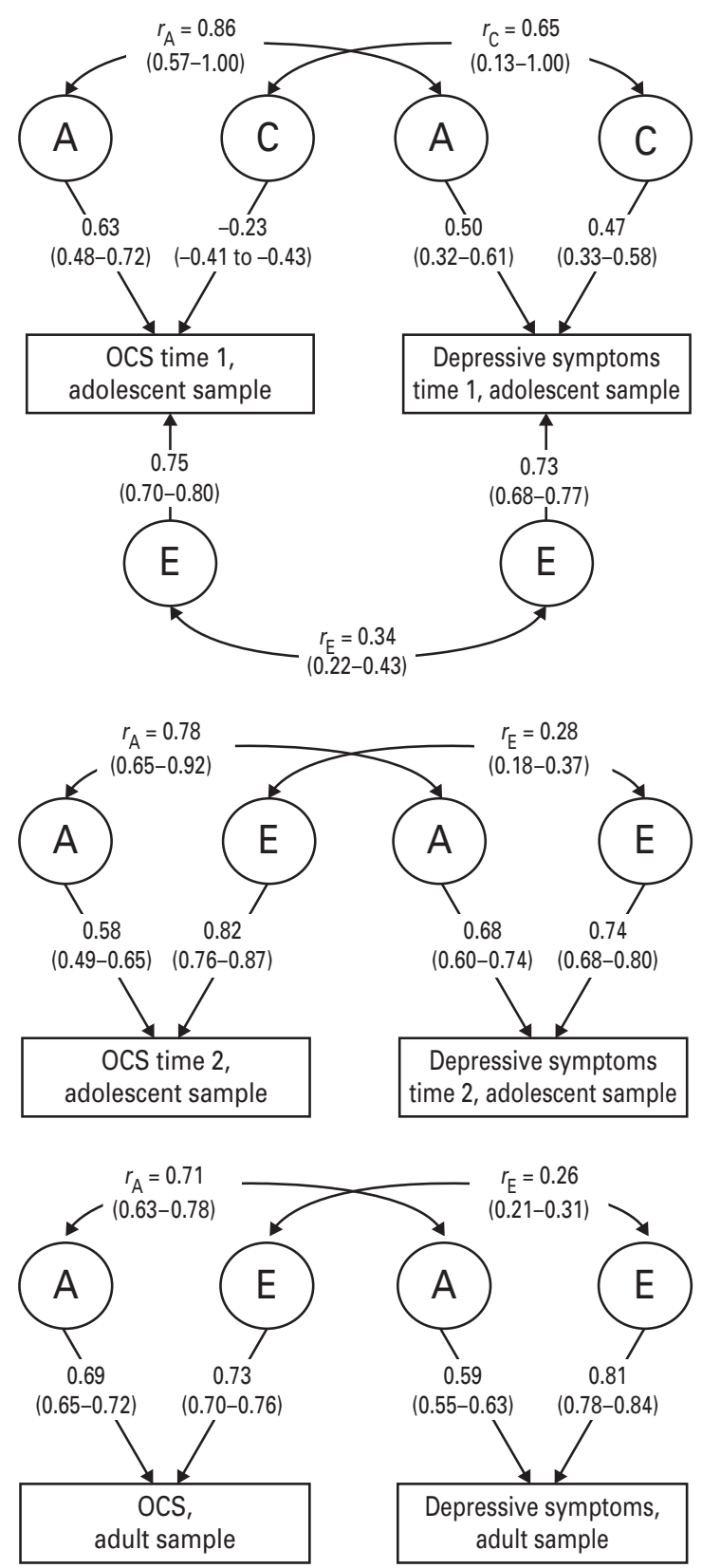

Fig. 3. Standardized unsquared path estimates of additive genetic (A), shared environmental $(\mathrm{C})$ and non-shared environmental (E) factors for the bivariate model of the association between obsessive-compulsive disorder symptoms (OCS) and depressive symptoms for three different age groups (from left to right: time 1 of the adolescent sample; time 2 of the adolescent sample; adult sample). Given are the genetic $\left(r_{\mathrm{A}}\right)$, shared environmental $\left(r_{\mathrm{C}}\right)$ and non-shared environmental $\left(r_{\mathrm{E}}\right)$ correlations between OCS and depressive symptoms. Values in parentheses are $95 \%$ confidence intervals.

Table 1 shows that these genetic correlations between OCS and depressive symptoms account for a similar proportion of the phenotypic correlation in each of the three age groups (52-65\%).
The shared environmental correlation between OCS and depressive symptoms was significant at time 1 in the adolescent sample, although the proportion of the phenotypic correlation explained by the shared environmental correlation was not.

The non-shared environmental correlation between OCS and depressive symptoms was significantly lower than the genetic correlation, and it was similar for the three age groups (range 0.26-0.34). Similarly, the proportion of the phenotypic correlation explained by common non-shared environmental factors was the same for the three age groups (35\%).

\section{Longitudinal genetic and environmental associations between OCS and depressive symptoms}

The longitudinal genetic relationship between OCS and depressive symptoms was explored in the adolescent sample using a multivariate Cholesky decomposition with four variables. A model of OCS at time 1 predicting later depressive symptoms was fitted, accounting for depressive symptoms at time 1 and OCS at time 2 (Fig. 1). An ACE model with scalars on OCS at time 1 and depressive symptoms at both time points provided the best fit (online Supplementary Table S5). Fig. 4 shows the results of the Cholesky decomposition. Fig. $4 a$ shows the additive genetic factors that explain the longitudinal association between OCS and depressive symptoms. Fig. $4 b, c$ shows to what extent the longitudinal association between OCS and depressive symptoms can be explained by overlap in shared and non-shared environmental factors, respectively.

Fig. 4 demonstrates that the genetic factors that account for depressive symptoms at time 1 also account for most of the genetic variance in subsequent depressive symptoms, and about half of the genetic variance in OCS at both time points. Similar to the cross-sectional twin modelling results, there was significant genetic overlap between OCS and depressive symptoms at time 1. After accounting for genetic variance shared with depressive symptoms at time 1 , the residual genetic variance in OCS at time 1 was not shared with depressive symptoms at time 2, but did account for some of the variance in OCS at time 2 .

Shared environmental factors had a significant effect on OCS at time 1 only. Non-shared environmental effects were largely trait-specific and time-specific, meaning that little of these effects were shared between variables. Specifically, after accounting for that shared with depressive symptoms at time 1 , there was no significant overlap in non-shared environmental effects between OCS at time 1 and depressive symptoms at time 2 . 

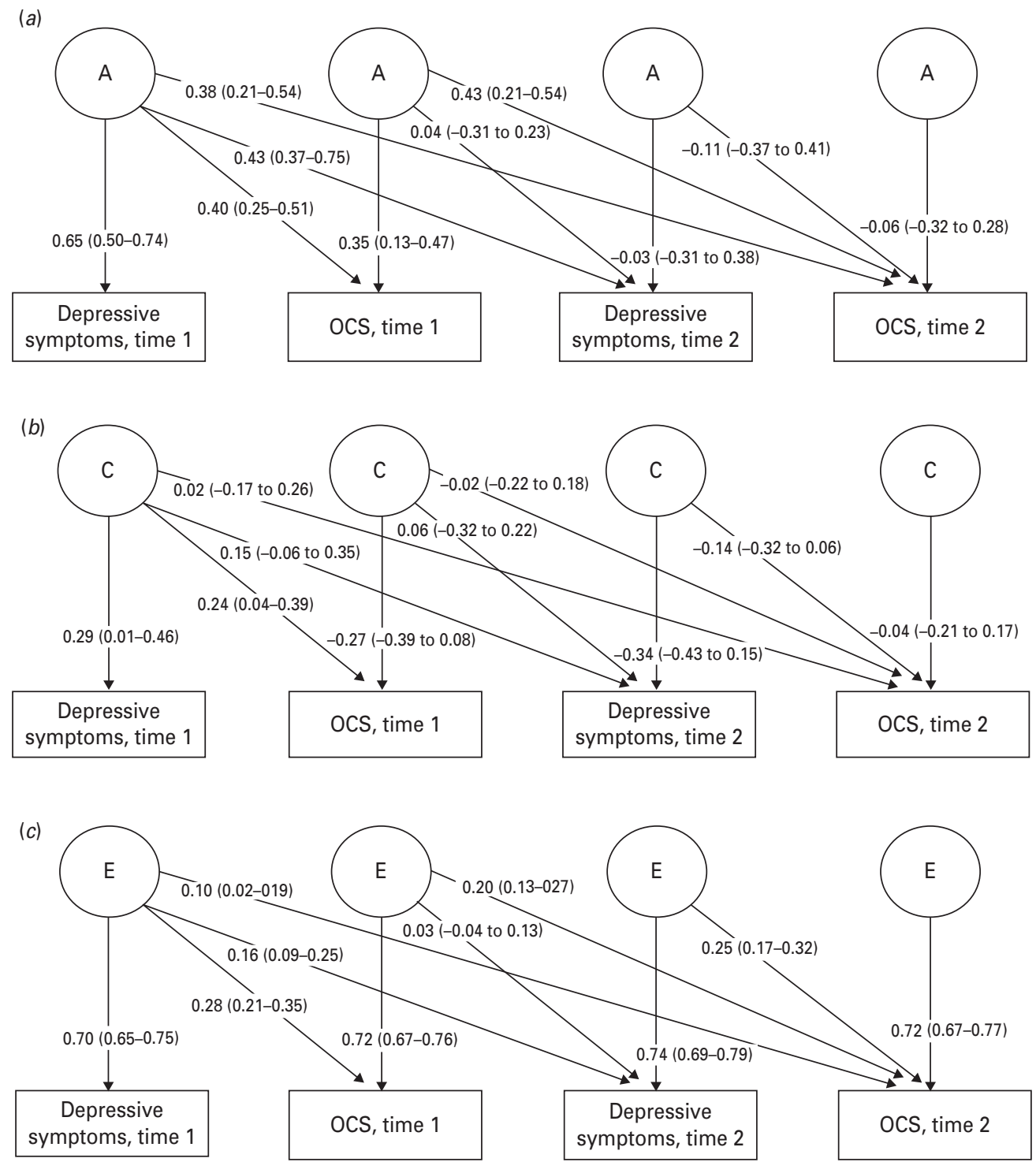

Fig. 4. Results from the Cholesky decomposition examining the longitudinal associations between obsessive-compulsive disorder symptoms (OCS) and depressive symptoms in the adolescent sample. The values presented are the standardized unsquared path estimates, with $95 \%$ confidence intervals in parentheses. (a) Associations between additive genetic factors (A) between the two traits. (b) Associations between shared environmental factors (C) between the two traits. (c) Path estimates of non-shared environmental factors (E).

\section{Discussion}

The present study tested two alternative hypotheses regarding the overlap between OCS and depressive symptoms. The first hypothesis was that depressive symptoms arise as a result of the burden associated with OCS. The second hypothesis was that the overlap between OCS and depressive symptoms could be explained by shared genetic factors. This study found support for the latter hypothesis. Each of the findings is discussed in turn.

The phenotypic results showed that OCS predicted later depressive symptoms to the same extent that depressive symptoms predicted later OCS. Univariate twin analyses demonstrated that OCS and depressive symptoms were moderately heritable in both adolescents and adults. The cross-sectional twin analyses showed that the genetic correlations between OCS and depressive symptoms were similarly high in both adolescents and adults. In the longitudinal twin analyses, the genetic and environmental factors associated with OCS at time 1 did not have a significant effect on later depressive symptoms, after controlling for the effects of depressive symptoms at time 1.

Consistent with previous studies in epidemiological and treatment-seeking samples of adults (Fireman et al. 
2001; Besiroglu et al. 2007; Ruscio et al. 2010) and young people (Douglass et al. 1995; Geller et al. 1996; Heyman et al. 2001), the present results showed a strong cross-sectional phenotypic relationship between OCS and depressive symptoms. The cross-sectional correlations between OCS and depressive symptoms were not identical from time 1 to time 2 in the adolescent sample, suggesting a weakening in their phenotypic associations over time. The within-trait across-time paths of OCS and depressive symptoms were far from 1 (0.48 and 0.44, respectively), which also suggested change over time for both traits. Longitudinal cross-lag model results showed that the significant relationship between OCS and depressive symptoms was of similar magnitude in both directions. This is similar to previous studies that demonstrated that the relationship between anxiety and depression was bi-directional (Moffitt et al. 2007; Zavos et al. 2012). To our knowledge this is the first study to demonstrate the bi-directionality of OCS and depressive symptoms.

Replicating previous studies, OCS and depressive symptoms were moderately heritable in both adolescents and adults (Kendler \& Gardner, 2011; Taylor, 2011; Zavos et al. 2012). With regard to the view that depressive symptoms are a functional consequence of OCS, the longitudinal analyses showed that there was no significant overlap in non-shared environmental factors between OCS and later depressive symptoms. This implies that the mechanism of evocative gene-environment correlation, where the genetically influenced behaviour of the individual (i.e. OCS) influences the likelihood of experiencing depressive symptoms (Jaffee \& Price, 2007; Kendler \& Baker, 2007), did not hold for the relationship between OCS and later depressive symptoms. This argues against the commonly held view that depressive symptoms arise secondarily to the burden caused by OCS (Peris et al. 2010; Storch et al. 2010, 2012; Anholt et al. 2011).

Instead, this study found support for the hypothesis that the strong association between OCS and depressive symptoms was explained through shared genetic factors. The cross-sectional twin analyses demonstrated a strong genetic correlation between OCS and depressive symptoms that was similar for adolescents and adults. The cross-sectional non-shared environmental correlations were far lower. Each phenotype seemed to have trait-specific environmental risks. This is consistent with studies showing that OCD and major depressive disorder co-aggregate in families (Nestadt et al. 2001; Carter et al. 2004, Hanna et al. 2011; Bienvenu et al. 2012; Goes et al. 2012) and suggests that the association between OCS and depressive symptoms is largely the result of genetic, rather than environmental, overlap. These results are similar to previous findings showing high genetic associations between anxiety and depression (Hettema, 2008; Kendler et al. 2008; Kendler \& Gardner, 2011; Zavos et al. 2012). Similar to other anxiety disorders, OCD may share certain personality traits with depression (e.g. neuroticism and negative affect), possibly explaining their phenotypic and genetic relationship (Hettema, 2008). However, there were also genetic effects unique to each of the phenotypes, a finding that has implications for future genesearching efforts.

The longitudinal twin analyses showed that the relationship between OCS and later depressive symptoms was largely explained through their crosssectional shared genetic factors. This is consistent with the hypothesis of genetic pleiotropy, whereby generalist genes largely explain the overlap between psychopathologies and environmental exposures primarily explain their differences (Eley, 1997; Lahey et al. 2011).

These findings are clinically important, as many patients presenting with OCS will also suffer from depressive symptoms, and vice versa. These findings showed that depressive symptoms should not be seen as a mere functional consequence of OCS; rather, the association was due to shared genetic effects. It is unclear what these shared effects might be, although they could represent heritable personality traits or cognitive styles (Lau et al. 2006; Hettema, 2008). These shared genetic risks might prove useful as potential targets for clinical interventions; indeed some of the existing therapies in the form of cognitive-behaviour therapy may be targeting such shared mechanisms (Zohar \& Insel, 1987; Anholt et al. 2011). Future research could focus on identifying such underlying mechanisms and investigating how they might be translated into treatment targets.

This study had a number of methodological strengths, such as the combined use of cross-sectional and longitudinal analyses and replication in two separate samples of different ages. There were also limitations. First, both OCS and depressive symptoms were investigated using different measures in the adolescent and adult samples, and their scores may as such measure slightly different constructs. However, the individual measures have been validated, which suggests that they were able adequately to capture the two phenotypes of OCS and depressive symptoms. Second, there was substantial attrition at follow-up in the adolescent sample. However, although attrition bias might complicate the estimation of trait prevalences, it is unlikely to affect the estimation of betweentrait associations (Wolke et al. 2009). Third, there were small differences in the univariate estimates of the genetic and environmental effects on OCS between those 
found in the adult analyses and those reported elsewhere using the same sample (Iervolino et al. 2011; Monzani et al. 2012). This is probably due to different approaches to the data, as the present study used transformed continuous data and the previous studies used categorized data. However, this does not translate into meaningful differences in terms of interpretation of the hypotheses, and analyses using categorized data lead to identical conclusions (data available upon request). Fourth, since only one time point of data was available, it was not possible to study the genetic and environmental influences on the longitudinal relationship between OCS and depressive symptoms in adults. However, our crosssectional analyses showed that the genetic correlation between OCS and depressive symptoms was similar for adolescents and adults.

These limitations notwithstanding, this is the first study to examine the genetic and environmental influences on both the cross-sectional and longitudinal relationship between OCS and depressive symptoms in adolescent and adult samples. The finding that OCS and depressive symptoms tend to co-occur primarily due to shared genetic factors offers a good explanation for the high co-morbidity between these disorders and also the fact that both disorders respond to similar pharmacological treatments. Future studies might focus on the mechanisms that underlie this genetic association between OCD and depressive symptoms, and use this knowledge to develop better treatments for these disabling disorders.

\section{Supplementary material}

For supplementary material accompanying this paper visit http://dx.doi.org/10.1017/S0033291713001591.

\section{Acknowledgements}

We thank the people and staff involved with the G1219 project and the Department of Twin Research (DTR) for their help and support in undertaking this project. Waves 1-3 of the G1219 study were supported by the W T Grant Foundation, the University of London Central Research Fund and a Medical Research Council Training Fellowship and Career Development Award (to T.C.E.). The DTR received support from the Wellcome Trust and the Department of Health via the National Institute for Health Research (NIHR) Comprehensive Biomedical Research Centre Award to Guy's \& St Thomas' NHS Foundation Trust in partnership with King's College London. A.S. acknowledges the support of the Wellcome Trust. T.A.M. acknowledges the support of the Leverhulme Trust (RPG-210).

\section{Declaration of Interest}

None.

\section{References}

Abramowitz JS, Deacon BJ (2006). Psychometric properties and construct validity of the Obsessive-Compulsive Inventory-Revised: replication and extension with a clinical sample. Journal of Anxiety Disorders 20, 1016-1035.

Angold A, Costello EJ, Messer SC, Pickles A, Winder F, Silver D (1995). The development of the short questionnaire for use in epidemiological studies of depression in children and adolescents. International Journal of Methods in Psychiatric Research 5, 237-249.

Anholt GE, Aderka IM, van Balkom AJ, Smit JH, Hermesh H, de Haan E, van Oppen P (2011). The impact of depression on the treatment of obsessive-compulsive disorder: results from a 5-year follow-up. Journal of Affective Disorders 135, 201-207.

Besiroglu L, Uguz F, Saglam M, Agargun MY, Cilli AS (2007). Factors associated with major depressive disorder occurring after the onset of obsessive-compulsive disorder. Journal of Affective Disorders 102, 73-79.

Bienvenu OJ, Samuels JF, Wuyek LA, Liang KY, Wang Y, Grados MA, Cullen BA, Riddle MA, Greenberg BD, Rasmussen SA, Fyer AJ, Pinto A, Rauch SL, Pauls DL, McCracken JT, Piacentini J, Murphy DL, Knowles JA, Nestadt G (2012). Is obsessive-compulsive disorder an anxiety disorder, and what, if any, are spectrum conditions? A family study perspective. Psychological Medicine 42, 1-13.

Boker S, Neale M, Maes H, Wilde M, Spiegel M, Brick T, Spies J, Estabrook R, Kenny S, Bates T, Mehta P, Fox J (2011). OpenMx: an open source extended structural equation modeling framework. Psychometrika 76, 306-317.

Carter AS, Pollock RA, Suvak MK, Pauls DL (2004). Anxiety and major depression comorbidity in a family study of obsessive-compulsive disorder. Depression and Anxiety 20, 165-174.

Costello EJ, Angold A (1988). Scales to assess child and adolescent depression: checklists, screens, and nets. Journal of the American Academy of Child and Adolescent Psychiatry 27, 726-737.

Costello EJ, Benjamin R, Angold A, Silver D (1991). Mood variability in adolescents: a study of depressed, nondepressed and comorbid patients. Journal of Affective Disorders 23, 199-212.

Douglass HM, Moffitt TE, Dar R, McGee R, Silva P (1995). Obsessive-compulsive disorder in a birth cohort of 18-year-olds: prevalence and predictors. Journal of the American Academy of Child and Adolescent Psychiatry 34, 1424-1431.

Eley T (1997). General genes: a new theme in developmental psychopathology. Current Directions in Psychological Science 6, 90-95.

Fireman B, Koran LM, Leventhal JL, Jacobson A (2001). The prevalence of clinically recognized obsessivecompulsive disorder in a large health maintenance 
organization. American Journal of Psychiatry 158, 1904-1910.

Flament MF, Whitaker A, Rapoport JL, Davies M, Berg CZ, Kalikow K, Sceery W, Shaffer D (1988). Obsessive compulsive disorder in adolescence: an epidemiological study. Journal of the American Academy of Child and Adolescent Psychiatry 27, 764-771.

Foa EB, Huppert JD, Leiberg S, Langner R, Kichic R, Hajcak G, Salkovskis PM (2002). The ObsessiveCompulsive Inventory: development and validation of a short version. Psychological Assessment 14, 485-496.

Geller DA, Biederman J, Griffin S, Jones J, Lefkowitz TR (1996). Comorbidity of juvenile obsessive-compulsive disorder with disruptive behavior disorders. Journal of the American Academy of Child and Adolescent Psychiatry 35, 1637-1646.

Gittleson NL (1966). Depressive psychosis in the obsessional neurotic. British Journal of Psychiatry 112, 883-887.

Goes FS, McCusker MG, Bienvenu OJ, Mackinnon DF, Mondimore FM, Schweizer B, Depaulo JR, Potash JB (2012). Co-morbid anxiety disorders in bipolar disorder and major depression: familial aggregation and clinical characteristics of co-morbid panic disorder, social phobia, specific phobia and obsessive-compulsive disorder. Psychological Medicine 42, 1449-1459.

Hanna GL, Himle JA, Hanna BS, Gold KJ, Gillespie BW (2011). Major depressive disorder in a family study of obsessive-compulsive disorder with pediatric probands. Depression and Anxiety 28, 501-508.

Hettema JM (2008). What is the genetic relationship between anxiety and depression? American Journal of Medical Genetics 148C, 140-146.

Heyman I, Fombonne E, Simmons H, Ford T, Meltzer H, Goodman R (2001). Prevalence of obsessive-compulsive disorder in the British nationwide survey of child mental health. British Journal of Psychiatry 179, 324-329.

Heyman I, Mataix-Cols D, Fineberg NA (2006). Obsessive-compulsive disorder. British Medical Journal 333, 424-429.

Iervolino AC, Rijsdijk FV, Cherkas L, Fullana MA, Mataix-Cols D (2011). A multivariate twin study of obsessive-compulsive symptom dimensions. Archives of General Psychiatry 68, 637-644.

Insel TR (1982). Obsessive compulsive disorder - five clinical questions and a suggested approach. Comprehensive Psychiatry 23, 241-251.

Ivarsson T, Melin K, Wallin L (2008). Categorical and dimensional aspects of co-morbidity in obsessivecompulsive disorder (OCD). European Child and Adolescent Psychiatry 17, 20-31.

Jaffee SR, Price TS (2007). Gene-environment correlations: a review of the evidence and implications for prevention of mental illness. Molecular Psychiatry 12, 432-442.

Kendell RE, Discipio WJ (1970). Obsessional symptoms and obsessional personality traits in patients with depressive illnesses. Psychological Medicine 1, 65-72.

Kendler KS, Baker JH (2007). Genetic influences on measures of the environment: a systematic review. Psychological Medicine 37, 615-626.
Kendler KS, Gardner CO (2011). A longitudinal etiologic model for symptoms of anxiety and depression in women. Psychological Medicine 41, 2035-2045.

Kendler KS, Gardner CO, Lichtenstein P (2008). A developmental twin study of symptoms of anxiety and depression: evidence for genetic innovation and attenuation. Psychological Medicine 38, 1567-1575.

Krebs G, Bolhuis K, Heyman I, Mataix-Cols D, Turner C, Stringaris A (2013). Temper outbursts in paediatric obsessive-compulsive disorder and their association with depressed mood and treatment outcome. Journal of Child Psychology and Psychiatry 54, 313-322.

Lahey BB, Van Hulle CA, Singh AL, Waldman ID, Rathouz PJ (2011). Higher-order genetic and environmental structure of prevalent forms of child and adolescent psychopathology. Archives of General Psychiatry 68, 181-189.

Lau JY, Rijsdijk F, Eley TC (2006). I think, therefore I am: a twin study of attributional style in adolescents. Journal of Child Psychology and Psychiatry 47, 696-703.

McAdams TA, Gregory AM, Rowe R, Zavos HMS, Barclay NL, Lau J, Maughan B, Eley TC (2013). The Genesis 12-19 (G1219) Study: a twin and sibling study of gene-environment interplay and adolescent development in the UK. Twin Research and Human Genetics 16, 134-143.

McKenzie N, Marks I (1999). Quick rating of depressed mood in patients with anxiety disorders. British Journal of Psychiatry 174, 266-269.

Moayyeri A, Hammond CJ, Hart DJ, Spector TD (2013). The UK Adult Twin Registry (TwinsUK Resource). Twin Research and Human Genetics 16, 144-149.

Moffitt TE, Harrington H, Caspi A, Kim-Cohen J, Goldberg D, Gregory AM, Poulton R (2007). Depression and generalized anxiety disorder: cumulative and sequential comorbidity in a birth cohort followed prospectively to age 32 years. Archives of General Psychiatry 64, 651-660.

Monzani B, Rijsdijk F, Iervolino AC, Anson M, Cherkas L, Mataix-Cols D (2012). Evidence for a genetic overlap between body dysmorphic concerns and obsessivecompulsive symptoms in an adult female community twin sample. American Journal of Medical Genetics 159B, 376-382.

Muthén LK, Muthén BO (1998). Mplus User's Guide. Muthén and Muthén: Los Angeles, CA.

Neale M, Cardon L (1992). Methodology for Genetic Studies of Twins and Families. Kluwer Academic: Dordrecht.

Nestadt G, Samuels J, Riddle MA, Liang KY, Bienvenu OJ, Hoehn-Saric R, Grados M, Cullen B (2001). The relationship between obsessive-compulsive disorder and anxiety and affective disorders: results from the Johns Hopkins OCD Family Study. Psychological Medicine 31, 481-487.

Peris TS, Bergman RL, Asarnow JR, Langley A, McCracken JT, Piacentini J (2010). Clinical and cognitive correlates of depressive symptoms among youth with obsessive compulsive disorder. Journal of Clinical Child and Adolescent Psychology 39, 616-626.

Rasmussen SA, Eisen JL (1992). The epidemiology and differential diagnosis of obsessive compulsive disorder. Journal of Clinical Psychiatry 53 (Suppl.), 5-10. 
Ruscio AM, Stein DJ, Chiu WT, Kessler RC (2010). The epidemiology of obsessive-compulsive disorder in the National Comorbidity Survey Replication. Molecular Psychiatry 15, 53-63.

Spence SH (1998). A measure of anxiety symptoms among children. Behavior Research and Therapy 36, 545-566.

Storch EA, Larson MJ, Muroff J, Caporino N, Geller D, Reid JM, Morgan J, Jordan P, Murphy TK (2010). Predictors of functional impairment in pediatric obsessive-compulsive disorder. Journal of Anxiety Disorders 24, 275-283.

Storch EA, Ledley DR, Lewin AB, Murphy TK, Johns NB, Goodman WK, Geffken GR (2006). Peer victimization in children with obsessive-compulsive disorder: relations with symptoms of psychopathology. Journal of Clinical Child and Adolescent Psychology 35, 446-455.

Storch EA, Lewin AB, Larson MJ, Geffken GR, Murphy TK, Geller DA (2012). Depression in youth with obsessive-compulsive disorder: clinical phenomenology and correlates. Psychiatry Research 196, 83-89.

Tambs K, Czajkowsky N, Roysamb E, Neale MC, Reichborn-Kjennerud T, Aggen SH, Harris JR, Orstavik RE, Kendler KS (2009). Structure of genetic and environmental risk factors for dimensional representations of DSM-IV anxiety disorders. British Journal of Psychiatry 195, 301-307.
Taylor S (2011). Etiology of obsessions and compulsions: a meta-analysis and narrative review of twin studies. Clinical Psychology Review 31, 1361-1372.

Van der Waerden BL (1933). Continuity theorem for semisimple lie groups [in German]. Mathematische Zeitschrift 36, 780-786.

Whiteside SP, Gryczkowski MR, Biggs BK, Fagen R, Owusu D (2012). Validation of the Spence Children's Anxiety Scale's obsessive compulsive subscale in a clinical and community sample. Journal of Anxiety Disorders 26, 111-116.

Wolke D, Waylen A, Samara M, Steer C, Goodman R, Ford T, Lamberts K (2009). Selective drop-out in longitudinal studies and non-biased prediction of behaviour disorders. British Journal of Psychiatry 195, 249-256.

Zavos HM, Rijsdijk FV, Eley TC (2012). A longitudinal, genetically informative, study of associations between anxiety sensitivity, anxiety and depression. Behavior Genetics 42, 592-602.

Zavos HM, Rijsdijk FV, Gregory AM, Eley TC (2010). Genetic influences on the cognitive biases associated with anxiety and depression symptoms in adolescents. Journal of Affective Disorders 124, 45-53.

Zohar J, Insel TR (1987). Obsessive-compulsive disorder: psychobiological approaches to diagnosis, treatment, and pathophysiology. Biological Psychiatry 22, 667-687. 